\title{
High-order dispersion effects in two-photon interference
}

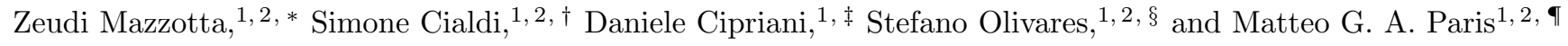 \\ ${ }^{1}$ Quantum Technology Lab, Dipartimento di Fisica, \\ Università degli Studi di Milano, I-20133 Milano, Italy \\ ${ }^{2}$ Istituto Nazionale di Fisica Nucleare, Sezione di Milano, Via Celoria 16, I-20133 Milan, Italy
}

(Dated: April 5, 2018)

\begin{abstract}
Two-photon interference and Hong-Ou-Mandel (HOM) effect are relevant tools for quantum metrology and quantum information processing. In optical coherence tomography, HOM effect is exploited to achieve high-resolution measurements with the width of the HOM dip being the main parameter. On the other hand, applications like dense coding require high-visibility performances. Here we address high-order dispersion effects in two-photon interference and study, theoretically and experimentally, the dependence of the visibility and the width of the HOM dip on both the pump spectrum and the downconverted photon spectrum. In particular, a spatial light modulator is exploited to experimentally introduce and manipulate a custom phase function to simulate the high-order dispersion effects.
\end{abstract}

PACS numbers: $42.50 . \mathrm{Ct}, 42.50 . \mathrm{Dv}$

\section{INTRODUCTION}

Two-photon interference (TPI) and in particular the the Hong-Ou-Mandel (HOM) 1 effect are relevant tools in photonic quantum technology. The HOM dip plays a central role in dense and superdense coding, since it allows to identify two of the four Bell states [2, 3]. Here the crucial parameter is the visibility of the HOM dip: the higher the visibility, the higher the mutual information [4, 5. TPI have found applications also in quantum metrology, where frequency entangled states of photons produced by downconversion (PDC) represent a resource for clock synchronization [6] and optical coherence tomography [7. However, the need to improve the resolution performances while using low-coherence interference leads to a halt: if one increases resolution widening the spectrum, the effect of dispersive media all along the path gets larger, thus preventing any further improvement 8 . Nevertheless, since the width of the HOM dip is not affected by dispersive media [9], TPI represents a promising candidate to face the resolution puzzle [17, 18]: the socalled Quantum Optical Coherence Tomography [10, 11] represents an interferometric technique for axial imaging offering several advantages over conventional methods.

This feature of the HOM effect, i.e. the independence of the dip width on dispersion, however, holds only if the pump laser is nearly monochromatic. When a broadband source is used to produce frequency entangled photons, the HOM dip becomes sensitive to even order dispersions [12, and both width and visibility are degraded.

In view of its relevance for quantum technology [9, 13,

\footnotetext{
*Electronic address: zeudi.mazzotta@unimi.it

${ }^{\dagger}$ Electronic address: simone.cialdi@unimi.it

${ }^{\ddagger}$ Electronic address: daniele.cipriani@mi.infn.it

$\S$ Electronic address: stefano.olivares@fisica.unimi.it

IElectronic address: matteo.paris@fisica.unimi.it
}

theoretical 14-16 and experimental [17 20] studies have been performed to characterize TPI and the HOM dip. However, a full analysis of both the visibility and the width of the HOM dip as a function of the pump spectrum and of the PDC spectrum is still missing.

The dispersive effects on the propagating photons can be described by a suitable phase function: this is the main topic of the present paper. In particular, we develop an experimental apparatus to introduce and manipulate a custom phase function with a spatial light modulator, as well as to work with different pump and PDC spectrum. As a matter of fact, we can simulate high-order dispersive effects of different transmission channels by exploiting the same setup. Indeed, we have performed a complete theoretical and experimental study of how the HOM effect is affected by the presence of second and third order dispersions, together with a direct comparison between theoretical and experimental results. The same setup can be used to compensate this kind of effect, paving the way for optimization in quantum technological applications involving PDC photons and optical fibers.

The paper is structured as follows. In Section II we review the description of frequency entangled photons obtained by PDC and provide the basic principles of twophoton interference in dispersive media. In Section III we describe in some details our experimental apparatus, whereas Section [V] is devoted to the experimental results. We discuss our findings in Section $\mathrm{V}$ and close the paper with some concluding remarks in Section VI.

\section{TWO-PHOTON INTERFERENCE}

The HOM effect occurs in TPI when two frequencyentangled photons generated by PDC interfere at a beam splitter (BS). In order to describe the TPI process after the propagation in dispersive media, we need to introduce the two-photon state produced via PDC and traveling from the source to the BS. In the presence of a broadband 
source laser the quantum state of the two photons, i.e. the signal " $s$ " and the idler " $i$ " photons, which interfere at the BS, is given by (see, e.g., [24]):

$$
\begin{aligned}
|\Phi\rangle=\iint & d \omega_{p} d \omega A\left(\omega_{p}\right) f(\omega) \\
& \times \mathrm{e}^{\mathrm{i} \phi_{s}\left(\omega_{p}, \omega\right)+\mathrm{i} \phi_{i}\left(\omega_{p},-\omega\right)}\left|\omega_{p}, \omega\right\rangle_{s}\left|\omega_{p},-\omega\right\rangle_{i}
\end{aligned}
$$

where $\omega_{p}$ and $\omega$ are the frequency shifts from the central pump and the central PDC frequencies, respectively, whereas $A\left(\omega_{p}\right)$ and $f(\omega)$ are the pump and the PDC spectral amplitudes, respectively. In Eq. (1) we introduced the two phase functions $\phi_{s}\left(\omega_{p}, \omega\right) \equiv \phi_{s}\left(\omega_{s}\right)$ and $\phi_{i}\left(\omega_{p}, \omega\right) \equiv \phi_{i}\left(\omega_{i}\right)$ denoting the phase functions induced by the propagation of the signal and the idler photon with frequencies $\omega_{s}=\left(\frac{\omega_{p}}{2}+\omega\right)$ and $\omega_{i}=\left(\frac{\omega_{p}}{2}-\omega\right)$, respectively. Since, in general, $\omega_{p} \ll \omega$, the effects of the broadband pump on $f(\omega)$ are negligible.

The phase functions are responsible for high-order effects on the propagation of state. In order to enlighten the different contributions, we expand $\phi_{x}\left(\omega_{x}\right)$ up to the third order in $\omega_{x}, x=s, i$, namely:

$$
\begin{aligned}
\phi_{x}\left(\omega_{x}\right) & \approx \sum_{k=0}^{3} \frac{1}{k !} \frac{\partial^{n}}{\partial \omega_{x}^{n}} \phi_{x}(0) \omega_{x}^{n} \\
& \approx \beta_{x}^{(0)}+\beta_{x}^{(1)} \omega_{x}+\frac{\beta_{x}^{(2)}}{2 !} \omega_{x}^{2}+\frac{\beta_{x}^{(3)}}{3 !} \omega_{x}^{3}
\end{aligned}
$$

where $\beta_{x}^{(k)}=\partial_{\omega_{x}}^{n} \phi_{x}(0)$. Each coefficient $\beta_{x}^{(k)}$ of the expansion plays a well defined role in the photon propagation (in our analysis we do not consider the constant phase $\beta_{x}^{(0)}$ since, as we will see, it will not contribute to the quantities of interest considered throughout the rest of the paper). The first order coefficient $\beta_{x}^{(1)}$ represents the "time" spent by the photons to reach the BS and is related to the group velocity. The second order coefficient $\beta_{x}^{(2)}$ refers to the group velocity dispersion experienced by both photons and it is due to the presence of dispersive media. Finally, the third order dispersion coefficient $\beta_{x}^{(3)}$, though it has usually a small impact in common materials, may have a huge effect when using very long optical fibers.

In each path, then, the two photons can experience a different dispersion, which may largely affect the TPI process and the occurrence of the HOM effect. More in details, if we introduce the "delay time" $\tau=\beta_{s}^{(1)}-\beta_{i}^{(1)}$, the probability of having coincident counts after the BS as a function $\tau$ reads:

$$
\begin{gathered}
P(\tau)=\iint d \omega_{p} d \omega\left|A\left(\omega_{p}\right)\right|^{2} \mid T f(\omega) \mathrm{e}^{\mathrm{i}\left[\phi_{s}\left(\omega_{p}, \omega\right)+\phi_{i}\left(\omega_{p},-\omega\right)\right]} \\
-\left.R f(-\omega) \mathrm{e}^{\mathrm{i}\left[\phi_{s}\left(\omega_{p},-\omega\right)+\phi_{i}\left(\omega_{p}, \omega\right)\right]}\right|^{2}
\end{gathered}
$$

where $T$ and $R$ are the transmission and reflection coefficients of the beam splitter.

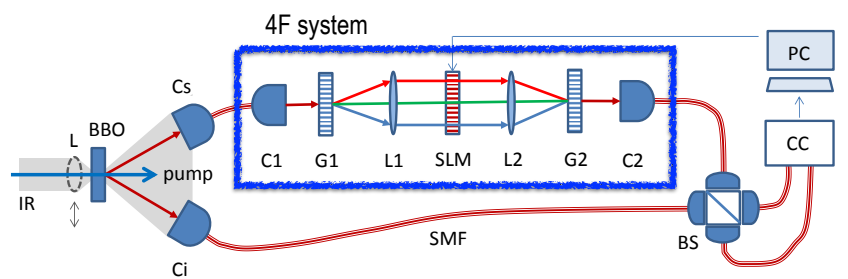

FIG. 1: Schematic diagram of our the two-photon interference apparatus. From left to right, the pump laser @ $405.5 \mathrm{~nm}$ (blue arrow) enters the BBO crystal and is downconverted. PDC photons are collected with couplers (Cs, Ci). The idler photon travels until the BS entirely through the fiber (SMF). The signal photon travels also through the $4 \mathrm{~F}$ optical system before entering again the fiber and reaching the BS. Two single photon detectors, with a Time-to-Amplitude Converter/Single-Channel Analyzer (TAC/SCA) system, detect the coincident counts (CC) at the two BS outputs. The shadowed region on the left, represents an IR laser beam that a lens (L) focalizes in the BBO position in order to perform an interference test. The See the text for details.

In the following we will show how we can experimentally manipulate and control the different contributions described above and, in particular, we will investigate their effects on the HOM dip and visibility.

\section{EXPERIMENTAL APPARATUS}

In Fig. 1 we show a schematic diagram of the experimental apparatus. The two frequency-entangled photon state is generated with a pump laser @ $405.5 \mathrm{~nm}$ by using a BBO crystal (1 mm thick). The signal and idler photons are then collected by two fiber couplers and sent into single spatial mode and polarization preserving fibers (SMF). A $1 \mathrm{~mm}$ thick crystal is enough to couple the maximum amounts of PDC radiation with our fiber couplers ( $\mathrm{Cs}$ and $\mathrm{Ci}$ ), as the interaction length inside the crystal between the pump and the fiber mode is slightly lower than $1 \mathrm{~mm}$ [21. The residual of the pump reaches a homemade high-resolution spectrometer (not shown in the figure). The possibility to stabilize the laser to different temperatures allows to keep the same central wavelength while changing the current (and, thus, the laser power) and also to perform two-photon interference measurements with different pump laser widths $(70 \mathrm{~mA}$ and $150 \mathrm{~mA}$ to have a narrower and broader pump respectively). When the idler photon enters the coupler $\mathrm{Ci}$, it travels entirely through the fiber towards the BS. Conversely, the signal photon, after a short fiber, enters a $4 \mathrm{~F}$ system [23], i.e. propagates in the air, through few optical devices (the couplers $\mathrm{C} 1$ and $\mathrm{C} 2$, the gratings G1 and G2 and lens L1 and L2) and a spatial light modulator (SLM). At the end of the $4 \mathrm{~F}$ system the signal photon is again coupled to a fiber and finally reaches the BS, where it interferes with the idler.

The SLM is a 1D liquid crystal mask (640 pixels, 
$100 \mu \mathrm{m} / \mathrm{pixel}$ ) and is placed on the Fourier plane between the two lenses L1 and L2 of the $4 \mathrm{~F}$ system (see Fig. 1). The SLM can be used to introduce and control a phase function $\phi_{\mathrm{M}}\left(\omega_{p}, \omega\right)$ on the signal photon. The possibility to choose at will $\phi_{\mathrm{M}}\left(\omega_{p}, \omega\right)$ allows us to achieve several goals (even at the same time): 1) to introduce from second to high-order dispersion effects on the HOM dip; 2) to perform a delay-time scan of the coincidence counts rate, avoiding any mechanical stress on the apparatus (this method to perform a delay scan is crucial for the stability of the $4 \mathrm{~F}$ coupling efficiency); 3) to compensate any dispersion effects introduced by the fibers and the $4 \mathrm{~F}$ system.

As a matter of fact, also the presence of the $4 \mathrm{~F}$ optical system on the signal path introduces a phase function $\phi_{4 \mathrm{~F}}\left(\omega_{p}, \omega\right)$ and, in particular, due to small shifts of the grating positions with respect to the optimal ones, it introduces second [22] and third order dispersions on its own.

Finally, the signal and idler optical fibers lead to the further phase functions $\phi_{\mathrm{f}, \mathrm{s}}\left(\omega_{p}, \omega\right)$ and $\phi_{\mathrm{f}, \mathrm{i}}\left(\omega_{p}, \omega\right)$, respectively.

Taking into account all the mentioned contributions, we have:

$$
\begin{aligned}
& \phi_{\mathrm{s}}\left(\omega_{p}, \omega\right)=\phi_{\mathrm{f}, \mathrm{s}}\left(\omega_{p}, \omega\right)+\phi_{4 \mathrm{~F}}\left(\omega_{p}, \omega\right)+\phi_{\mathrm{M}}\left(\omega_{p}, \omega\right), \\
& \phi_{\mathrm{i}}\left(\omega_{p}, \omega\right)=\phi_{\mathrm{f}, \mathrm{i}}\left(\omega_{p}, \omega\right),
\end{aligned}
$$

and we can rewrite Eq. (4) as:

$$
\begin{aligned}
P(\tau)= & \iint d \omega_{p} d \omega\left|A\left(\omega_{p}\right)\right|^{2}\left\{T^{2} f(\omega)^{2}+R^{2} f(-\omega)^{2}\right. \\
& \left.-2 R T \Re \mathrm{e}\left[f(\omega) \overline{f(-\omega)} \mathrm{e}^{\mathrm{i} \phi_{\mathrm{tot}}\left(\omega_{p}, \omega\right)}\right]\right\}, \\
\approx & \iint d \omega_{p} d \omega\left|A\left(\omega_{p}\right)\right|^{2}\left\{T^{2} f(\omega)^{2}+R^{2} f(-\omega)^{2}\right. \\
& -2 R T \Re \mathrm{e}[f(\omega) \overline{f(-\omega)} \\
& \left.\left.\times \mathrm{e}^{\mathrm{i} 2 \omega \tau+i \phi_{\mathrm{tot}}^{(2)}\left(\omega_{p}, \omega\right)+i \phi_{\mathrm{tot}}^{(3)}\left(\omega_{p}, \omega\right)}\right]\right\},
\end{aligned}
$$

where $\phi_{\text {tot }}\left(\omega_{p}, \omega\right)=\phi_{\mathrm{s}}\left(\omega_{p}, \omega\right)-\phi_{\mathrm{s}}\left(\omega_{p},-\omega\right)+\phi_{\mathrm{i}}\left(\omega_{p},-\omega\right)-$ $\phi_{\mathrm{i}}\left(\omega_{p}, \omega\right)$, and the total second and third order dispersion terms are (note that, as mentioned, the zero order terms cancel out):

$$
\begin{aligned}
& \phi_{\text {tot }}^{(2)}\left(\omega_{p}, \omega\right)=\beta_{\text {tot }}^{(2)} \omega_{p} \omega, \\
& \phi_{\text {tot }}^{(3)}\left(\omega_{p}, \omega\right)=\frac{1}{4} \beta_{\text {tot }}^{(3)} \omega_{p}^{2} \omega+\frac{1}{3} \beta_{\text {tot }}^{(3)} \omega^{3},
\end{aligned}
$$

respectively, with:

$$
\beta_{\mathrm{tot}}^{(k)}=\beta_{\mathrm{f}}^{(k)}+\beta_{4 \mathrm{~F}}^{(k)}+\beta_{\mathrm{M}}^{(k)} \quad(k=2,3) .
$$

According to our formalism, the quantities appearing at the RHS of the last equation refer to the $k$-th order dispersion coefficients due to the fibers (f), to the $4 \mathrm{~F}$ system $(4 \mathrm{~F})$ and to the SLM (M). It is worth noting that $\beta_{\mathrm{f}}^{(k)}=d_{\mathrm{f}}^{(k)} \Delta L$, where $d_{\mathrm{f}}^{(k)}$ is the $k$-th order dispersion of the fiber and $\Delta L$ is the length difference between the signal and idler optical fiber.

It is straightforward to see that in the presence of an almost monochromatic pump, i.e., $\omega_{p} \approx 0$, the only relevant dispersion effect comes from the second term of the RHS in Eq. (9), that is the second order dispersion does not affect the HOM dip shape.

The presence of several optical components in the apparatus leads to an overall loss in purity of the polarization quantum state of the photons. In order to assess the purity, we performed an interferometric measurement: after a strong filtering with neutral density filters, we focus a $811 \mathrm{~nm}$ laser in the $\mathrm{BBO}$ position and couple the radiation of the resulting divergent beam with the fibers. Then we obtain a Mach-Zehnder interferometer where the action of focusing plays the role of the first BS. Counts are then balanced by carefully positioning the focusing lens: this is crucial in order to have the same amount of direct counts on both paths, thus maximizing the interferogram visibility.

If $\theta$ is the polarization mismatching between the two beams reaching the BS, the visibility of the interferogram is given by $V_{\mathrm{I}}=|\cos \theta|$. It is now straightforward to show that the visibility of the HOM dip $V_{\mathrm{H}}$ is directly linked to the interferogram visibility by

$$
V_{\mathrm{H}}=\frac{R T V_{\mathrm{I}}^{2}}{1-2 R T-R T V_{\mathrm{I}}^{2}} .
$$

In our experiment the measured visibility is $(95.6 \pm 0.5) \%$, corresponding to a mismatching angle of $\theta=(17.2 \pm 0.9)^{\circ}$ and a purity factor $p=\cos ^{2} \theta \approx 91 \%$. Since, given $p$, the coincidence counts probability becomes:

$$
P_{\text {tot }}(\tau)=P(\tau) p+\frac{1}{2}(1-p),
$$

then the maximum dip visibility we can achieve with the measured $T=0.467, R=0.533$ and a symmetric PDC spectrum, i.e. $f(\omega)=f(-\omega)$, turns out to be $(82 \pm 2) \%$.

In Fig. 2 we show the pump and PDC spectra we used during our measurements. On the pump side, in Fig. 2(a) one can see the difference in the pump spectrum width between two different current settings: the narrower spectrum $\left(\Delta_{\mathrm{p}} \approx 0.5 \mathrm{~nm}\right)$ corresponds to a current of $70 \mathrm{~mA}$, while a current of $150 \mathrm{~mA}$ leads to a broader spectrum $\left(\Delta_{\mathrm{p}} \approx 1 \mathrm{~nm}\right)$. We measured the pump spectrum with a homemade spectrometer as follows. We collected the residual of the pump light with a fiber and delivered it towards a collimating lens. Then, a grating $(3600 \mathrm{l} / \mathrm{mm})$ divides the collimated beam into its spectral components. These are then focused with a $300 \mathrm{~mm}$ lens onto the sensor of a CMOS camera placed on the Fourier plane.

Figure 2(b) shows the PDC spectrum. We can choose the nominal spectrum $\left(\Delta_{\mathrm{PDC}}=20 \mathrm{~nm}\right)$ or the "cut" spectrum $\left(\Delta_{\mathrm{PDC}}=10 \mathrm{~nm}\right)$ with a slit placed on the Fourier plane of the $4 \mathrm{~F}$ system. We measured PDC spec- 


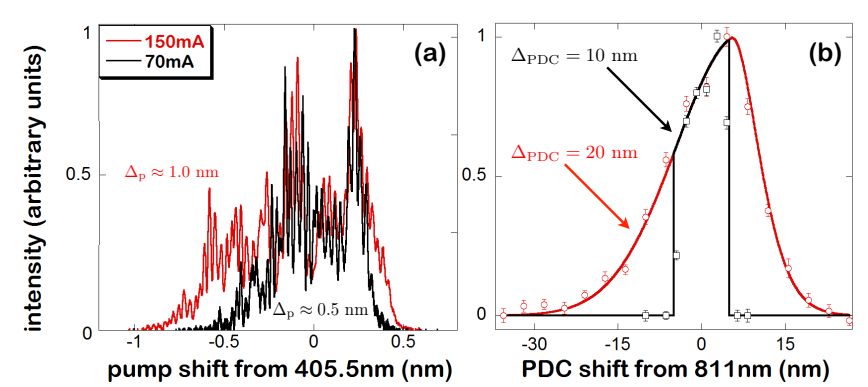

FIG. 2: Pump and PDC spectra. Panel (a): measured pump spectrum shift about the central wavelength of $405.5 \mathrm{~nm}$, with $70 \mathrm{~mA}$ (spectrum width $\Delta_{\mathrm{p}} \approx 1.0 \mathrm{~nm}$ ) and $150 \mathrm{~mA}$ (spectrum width $\Delta_{\mathrm{p}} \approx 0.5 \mathrm{~nm}$ ) current. Panel (b): PDC spectrum data points and curve fit, with a slit on the Fourier plane (spectrum width $\Delta_{\mathrm{PDC}}=10 \mathrm{~nm}$ ) and without it (spectrum width $\Delta_{\mathrm{PDC}}=20 \mathrm{~nm}$ ).

tra with a $2 \mathrm{~mm}$ slit on the Fourier plane of the $4 \mathrm{~F}$ system. We calibrated the slit using a graduated reference on the Fourier plane: we found the $811 \mathrm{~nm}$ component position for the slit, we measured a dispersion coefficient of $3.62 \mathrm{~nm}$ for a $2 \mathrm{~mm}$ slit displacement and finally, in order to measure the spectrum, for each slit position (and therefore for each wavelength) we recorded coincidence counts from the detectors.

As mentioned above, one usually desires to have symmetric PDC spectra. However, Fig. 2(b) shows that we used a non symmetric signal photon spectrum. It is worth noting that our apparatus allows us to center the PDC spectra on $811 \mathrm{~nm}$ : this is possible by transversally moving a mirror that delivers the pump inside the BBO crystal. In this way we make the pump axis (that is the PDC cone axis) be at the exactly same distance from both fiber couplers. However, we found that the $4 \mathrm{~F}$ system introduced an asymmetric cut on the PDC spectrum tails, that is translated in an antisymmetric cut on the idler photon spectrum. This makes the two photons distinguishable and the visibility worsen. The spectrum we report in Fig. 2(b) correspond to the spectrum giving the highest visibility possible in our configuration: the peaks are not superimposed at $811 \mathrm{~nm}$ but the tails are. Overall, this makes the two photons less distinguishable than the situation where the tails are not superimposed.

\section{EXPERIMENTAL RESULTS}

A slit placed under one of the fiber couplers is used to match signal and idler paths. The SLM delay-time scan method allows us to match the two paths with an error of less than $1 \mu \mathrm{m}$. A range of several picoseconds can be covered with an SLM delay-time scan. Therefore, before measuring, signal and idler paths were always perfectly matched. In Fig. 3, we report some measured dip profiles (points) together with the corresponding theoretical predictions (lines). It is worth noting that the predictions
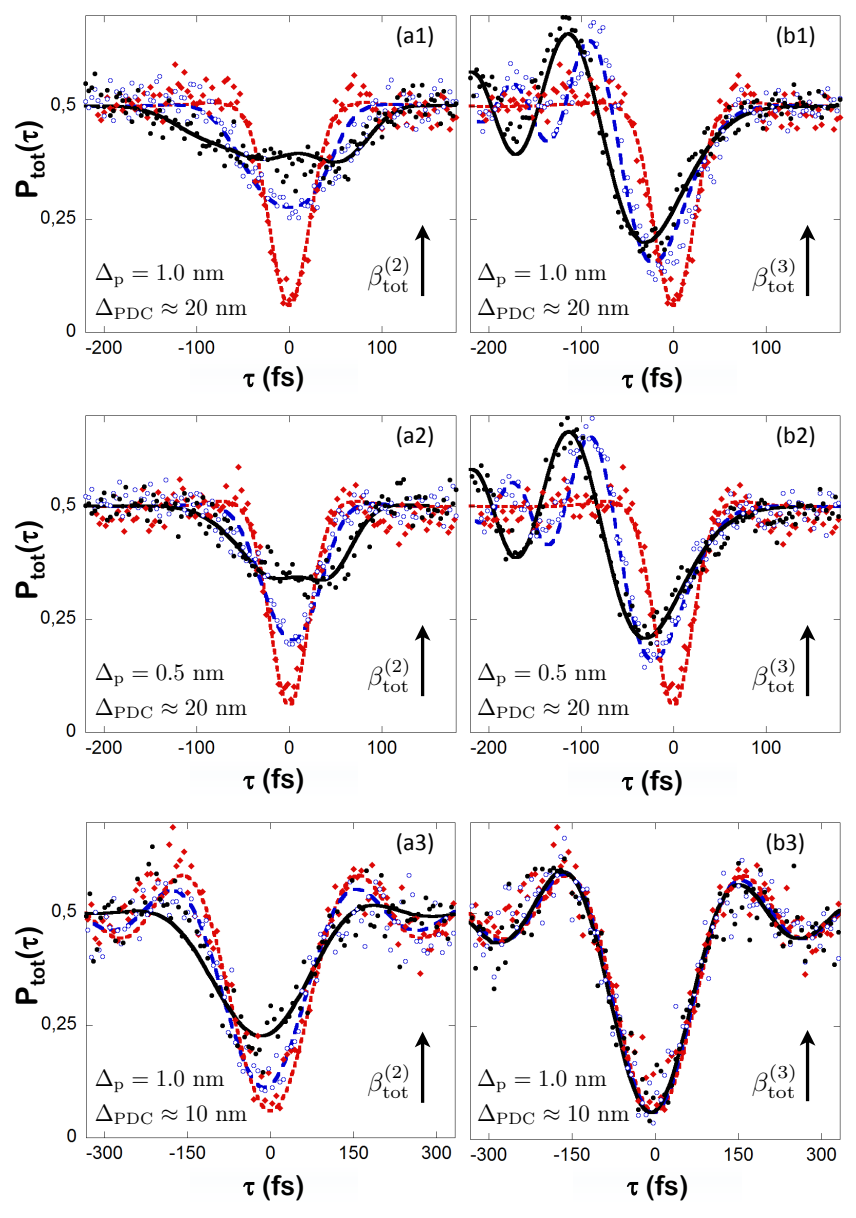

FIG. 3: The HOM dip in the presence of second and third order dispersion. In panels (a1), (a2), and (a3) we show the dip profiles for three different second order dispersion values, from the lower to the higher dip we set $\beta_{\text {tot }}^{(2)}=0 \mathrm{fs}^{2}$ (red dotted line and rhombus for theoretical prevision and data points, respectively), $17.6 \times 10^{3} \mathrm{fs}^{2}$ (blue dashed line and empty circles), $35.2 \times 10^{3} \mathrm{fs}^{2}$ (black solid line and filled circles). In panels (b1), (b2), and (b3) we show the dip profiles for three different third order dispersion values, from the lower to the higher dip we set $\beta_{\text {tot }}^{(3)}=0 \mathrm{fs}^{3}$ (red dotted line and rhombus), $17.6 \times 10^{4} \mathrm{fs}^{3}$ (blue dashed line and empty circles), $35.2 \times 10^{4} \mathrm{fs}^{3}$ (black solid line and filled circles). The corresponding values of $\Delta_{\mathrm{p}}$ and $\Delta_{\mathrm{PDC}}$ of the pump and PDC spectrum widths are reported in the panels. All solid lines are theoretical predictions obtained from Eq. 12 .

are obtained from Eq. 12 and from the experimental spectra of Fig. 2 without any fitting parameter. The experimental data are normalized using the mean value at high delay time (i.e., outside the HOM dip). The time windows for the counts acquisition for each point are $2 \mathrm{~s}$ and $2.5 \mathrm{~s}$, respectively, for the cases at $150 \mathrm{~mA}$ and $70 \mathrm{~mA}$.

Each line in Fig. 3 corresponds to a different configuration. On the left column, we show three different profiles related to three different values of the second order dispersion coefficient; on the right column, we re- 
port the profiles for three different values of the third order dispersion coefficient. In the top panels, (a1) and (b1), we have results for the broader pump spectrum $(1 \mathrm{~nm})$ and normal PDC spectrum $(20 \mathrm{~nm})$ (from now on we refer to these values as to configuration S1). We found a huge dependence on second and third order dispersion: the dip visibility decreases and its width increases, with increasing dispersion. In the panels (a2) and (b2), we show results for the case of the narrower pump spectrum $\left(\Delta_{\mathrm{p}} \approx 0.5 \mathrm{~nm}\right)$ and normal PDC spectrum $\left(\Delta_{\mathrm{PDC}}=20 \mathrm{~nm}\right.$, configuration $\left.\mathrm{S} 2\right)$. In this case, the dependence of the dip shape on the second order dispersion is significantly weaker, while the dependency on the third order dispersion remains the same of the previous case. This is in agreement with the fact that two-photon interference is affected by second order dispersion only if the pump is not monochromatic, while it is always affected by third order dispersion (and all odd orders, including the first order).

In the above cases, one may quantify the effects of the third order dispersion on the HOM dip by looking at the visibility. On the bottom line of Fig. 3, panels (a3) and (b3), we have the case of the broader pump spectrum $\left(\Delta_{\mathrm{p}} \approx 1.0 \mathrm{~nm}\right)$ and the cut PDC spectrum $\left(\Delta_{\mathrm{PDC}}=10 \mathrm{~nm}\right.$, configuration S3). While we still have a visible dependence on the second order dispersion, the dependence on the third order dispersion disappears. The width of the compensated dip, here, is much larger than in the cases above. This comes directly from Eq. (7). A peculiar behaviour can be seen here: symmetric oscillation appear on the dip, caused by the clean cut of the PDC spectrum.

\section{DISCUSSION}

As we mentioned in the introduction, by a suitable choice of the phase function we can simulate the high order dispersion effects of a particular transmission channel. In particualr, the values of the second and third order contribution we used in our experiment are related to those obtained in current optical fibers. For instance, a dispersion of $30 \times 10^{3} \mathrm{fs}^{2}$ is induced by a fused silica fiber $\approx 850 \mathrm{~mm}$ long $\left(d_{\mathrm{f}}^{(2)} \approx 35 \mathrm{fs}^{2} / \mathrm{mm}\right)$. For a third order dispersion of $300 \times 10^{3} \mathrm{fs}^{3}$, on the other hand, $\approx 11 \mathrm{~m}$ of fiber are needed $\left(d_{\mathrm{f}}^{(3)} \approx 30 \mathrm{fs}^{3} / \mathrm{mm}\right)$ or, for example, $35 \mathrm{~cm}$ of ZnSe $\left(d_{\mathrm{f}}^{(3)}=870 \mathrm{fs}^{3} / \mathrm{mm}\right)$. It is worth noting that these lengths are quite small compared to those to be used in telecommunications. For this reason the full comprehension of these dispersion effects on the HOM dip becomes a fundamental a prerequisite.

In Fig. 4 we summarize our main results: we can see the behaviour of the dip visibility and full width at half maximum (FWHM) as a function of second and third order dispersions. The visibility and the FWHM are defined with respect to the normalized value and are obtained by using suitable fitting functions. Each plot
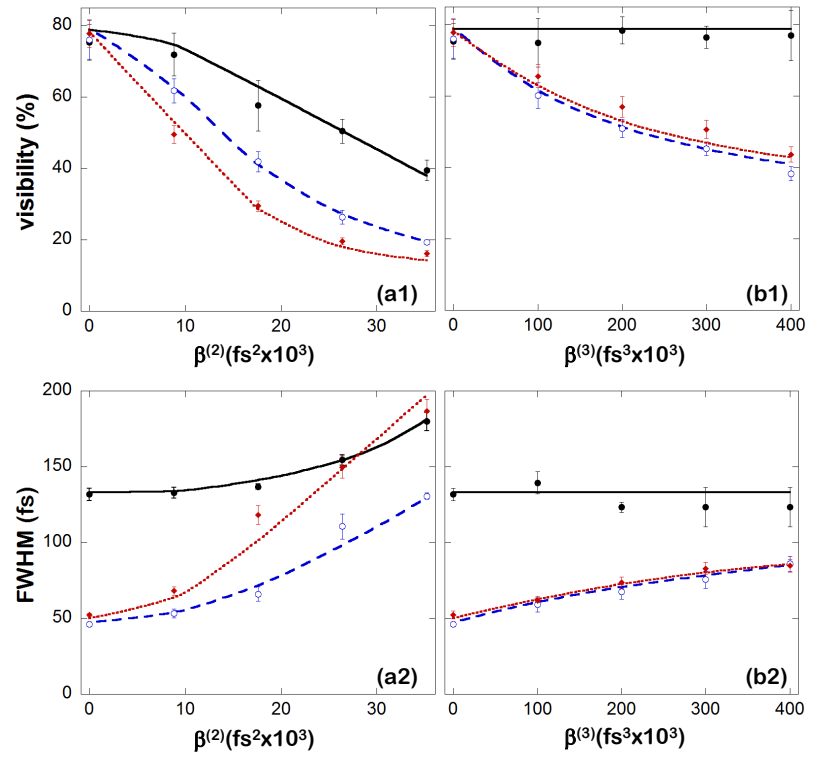

FIG. 4: Visibility and FWHM of the HOM dip as a function of $\beta_{\mathrm{tot}}^{(2)}$ and $\beta_{\mathrm{tot}}^{(3)}$ for different pump and PDC spectra: PDC $\Delta_{\mathrm{PDC}}=10 \mathrm{~nm}$ and $\Delta_{\mathrm{p}}=1.0 \mathrm{~nm}$ (solid lines); Dotted line: $\Delta_{\mathrm{PDC}}=20 \mathrm{~nm}$ and $\Delta_{\mathrm{p}}=1.0 \mathrm{~nm}$ (dotted); $\Delta_{\mathrm{PDC}}=20 \mathrm{~nm}$ and $\Delta_{\mathrm{p}}=0.5 \mathrm{~nm}$ (dashed lines).

reports the results for the three different experimental configurations introduce above: dotted red line for S1, dashed blue line for S2 and solid black line for S3. In panel (a1), on the upper left, we see how visibility decreases as the the second order dispersion increases. This worsening gets less sharp if we cut down either the PDC spectrum (S3) or the pump spectrum (S2). In panel (a2), on the lower left, we see how the dip FWHM increases (the dip gets broader) when the second order dispersion coefficient increases. As already mentioned above, without dispersion the dip FWHM in S3 is larger than in S1 and S2. In both S2 and S3, the FWHM is much less sensitive to the second order dispersion compared to the case of broad pump and PDC spectra.

In Fig. 4(b1) and (b2), on the right, we studied again the changes in the dip visibility and FWHM as a function of the third order dispersion. We can see how the cut of the PDC spectrum keeps frozen the dip parameters, making them insensitive to any third order dispersion coefficient. However, we can also clearly see that the visibility and the FWHM do not depend on the pump spectrum.

\section{CONCLUSONS}

In this paper we have designed and developed an experimental setup to simulate second and third order dispersion effects on the propagation of PDC photons. By using our innovative apparatus, which is able to introduce and manipulate a custom phase function with a SLM, we 
have analyzed high-order dispersion effects in two-photon interference. In particular, we have studied theoretically and experimentally, the dependence of both the visibility and the width of the HOM dip on both the pump spectrum and on the downconverted photon spectrum.

Though we have mostly used our system to introduce high-order dispersion effects in the propagation of the states, the same setup can be used to compensate such effect. Therefore, on the one hand, our results clarify the role of the different dispersion coefficients and, on the other hand, pave the way for optimization procedures in communications protocols and other applications in quantum technology involving PDC photons and optical fibers.

\section{Acknowledgments}

This work has been supported by UniMI through the UNIMI14 grant 15-6-3008000-609 and the H2020 Transition Grant 15-6-3008000-625, and by EU through the collaborative project QuProCS (Grant Agreement 641277).
[1] C. K. Hong, Z. Y. Ou, and L. Mandel, Phys. Rev. Lett. 59, 2044 (1987).

[2] H. Weinfurter, Europhys. Lett. 25, 559 (1994)

[3] S. L. Braunstein, and A. Mann, Phys. Rev. A 51, R1727 (1995).

[4] K. Mattle, H. Weinfurter, P. G. Kwiat, and A. Zeilinger, Phys. Rev. Lett. 76, 4656 (1996).

[5] J. T. Barreiro, T.-C. Wei, P. G. Kwiat, Nature 4, 282 (2008).

[6] V. Giovannetti, S. Lloyd, L. Maccone, and F. N. C. Wong Phys. Rev. Lett. 87, 117902 (2001).

[7] D. Huang, E. A. Swanson, C. P. Lin, J. S. Schuman, W. G. Stinson, W. Chang, M. R. Hee, T. Flotte, K. Gregory, C. A. Puliafito, and J. G. Fujimoto, Science 254, 1178 (1991).

[8] A. G. Van Engen, S. A. Diddams, and T. S. Clement, Appl. Opt. 37, 5679 (1998).

[9] A. M. Steinberg, P. G. Kwiat, R. Y. Chiao, Phys. Rev. Lett. 68, 2421 (1992).

[10] A. F. Abouraddy, M. B. Nasr, B. E. A. Saleh, A. V. Sergienko, and M. C. Teich, Phys. Rev. A 65, 053817 (2002).

[11] M. B. Nasr, B. E. A. Saleh, A. V. Sergienko, and M. C. Teich, Phys. Rev. Lett. 91, 083601 (2003).

[12] K. J. Resch, R. Kaltenbaek, R. Lavoie, and D. N. Biggerstaff, Proc. SPIE 7465, 74650N (2009).
[13] K. Mattle, H. Weinfurter, P. G. Kwiat, and A. Zeilinger Phys. Rev. Lett. 76, 4656 (1996).

[14] B. Dayan, Phys. Rev. A. 76, 043813 (2007).

[15] J. Peřina, Jr., A. V. Sergienko, B. M. Jost, B. E. A. Saleh, and M. C. Teich, Phys. Rev. A 59, 2359 (1999).

[16] R. Erdmann, D. Branning, W. Grice, and I. A. Walmsley, Phys. Rev. A 62, 053810 (2000).

[17] M. Okano, R. Okamoto, A. Tanaka, S. Ishida, N. Nishizawa, and S. Takeuchi, Phys. Rev. A 88, 043845 (2013).

[18] M. Okano, H. H. Lim, R. Okamoto, N. Nishizawa, S. Kurimura, and S. Takeuchi, Sc. Rep. 5, 18042 (2015).

[19] M. Atatüre, A. V. Sergienko, B. M. Jost, B. E. A. Saleh, and M. C. Teich, Phys. Rev. Lett. 83, 1323 (1999).

[20] R. Okamoto, S. Takeuchi, and K. Sasaki, Phys. Rev. A. 74, 011801(R) (2006).

[21] L. E. Vicent, A. B. U'Ren, R. Rangarajan, C. I Osorio, J. P Torres, L. Zhang, and I. A Walmsley, New J. Phys. 12, 093027 (2010).

[22] S. Cialdi, C. Vicario, M. Petrarca, and P. Musumeci, Appl. Opt. 46, 4959 (2007).

[23] A. M. Weiner, Rev. Sci. Instrum. 71, 1929 (2000).

[24] A. Smirne, S. Cialdi, G. Anelli, M. G. A. Paris, and B. Vacchini Phys. Rev. A 88, 012108 (2013). 